\title{
Exposición de resultados de aprendizaje: metodologías y estrategia para promocionar el perfil profesional y la continuidad en la carrera
}

\section{Exhibition of learning outcomes: methodologies and strategy to promote professional profile and career continuity}

\author{
Paola Cristina Toapanta-Pinta ${ }^{1}$; Mercy Dolores Rosero-Quintana ${ }^{2}$; \\ Sara Rosa Céspedes-Granda ${ }^{3}$; Santiago Noé Vasco-Morales ${ }^{4}$ \\ \{ptoapanta@uce.edu.ec; mdrosero@uce.edu.ec; \\ scespedes@uce.edu.ec; snvasco@uce.edu.ec\}
}

Fecha de recepción: 6 de junio de 2020 - Fecha de aceptación: 12 de julio de 2020

\begin{abstract}
Resumen: La exposición de resultados de aprendizaje a los alumnos que inician su formación a través de distintas metodologías podría mejorar la tasa de retención de los estudiantes. Muchos estudiantes ingresan a una carrera que no es su primera elección y esto interfiere con la motivación para continuar su formación académica. Se deben generar estrategias que mejoren la afinidad de los estudiantes hacia las competencias de la profesión, por tanto se organizó la semana de presentación de resultados de aprendizaje de fin del semestre; $y$, con el objetivo de conocer la percepción de los estudiantes sobre las metodologías y contenidos utilizados durante la semana y su eficacia para demostrar el rol del profesional de obstetricia y motivar la continuidad de estudiantes de primer semestre en la carrera, se realizó un estudio transversal descriptivo y analítico a través de una encuesta en escala Likert, aplicada a los estudiantes que asistieron a las actividades realizadas durante la semana y el uso de modelos Bayesianos ordinales. Los resultados de la percepción de los estudiantes demuestran calificaciones positivas: foro con intervención de expertos 90,5\%, exposición magistral 89,4\%, casa abierta 83,6\%, sociodrama $90 \%$ y simulación $93,1 \%$. El 90,4\% de estudiantes de la carrera indicó que los temas presentados demostraron el rol profesional, mientras que el 84,3\% de alumnos de primer semestre dijo sentirse motivado a continuar sus estudios dentro de la carrera. Se concluye que la percepción sobre las metodologías utilizadas en la semana de exposición de resultados de aprendizaje es positiva y a través de ellas se logró demostrar el rol profesional y motivar a los estudiantes de primer semestre para continuar su formación en la carrera.
\end{abstract}

Palabras clave - Educación médica, pregrado, motivación, didáctica.

\footnotetext{
${ }^{1}$ Doctora en Medicina y Cirugía, Máster en Pediatría y Neonatología. Universidad Central del Ecuador.

${ }^{2}$ Obstetriz, Magíster en Adolescencia.

Universidad Central del Ecuador.

${ }^{3}$ Obstetriz, Magíster en Gerencia Clínica en Salud Sexual y Reproductiva. Universidad Central del Ecuador.

${ }^{4}$ Doctor en Medicina y Cirugía, Especialista en Pediatría.

Universidad Central del Ecuador.
}

Cómo citar: Toapanta-Pinta, P. C., Rosero-Quintana, M. D., Céspedes-Granda S. R., \& Vasco-Morales, S. N. (2020). Exposición de resultados de aprendizaje: metodologías y estrategia para promocionar el perfil profesional y la continuidad en la carrera. Magazine De Las Ciencias: Revista De Investigación E Innovación, 5(7), 83-92. https://revistas.utb.edu.ec/index.php/magazine/article/view/925 


\begin{abstract}
The exposition of learning outcomes to students starting their training through different methodologies could being improved the retention rate of students. Many students are studing a career that was not their first choice; this interferes with their motivation to continue their academic training. Strategies should be generated that improve the affinity of students towards the competences of the profession, therefore the week of learning results presentation at the end of the semester was organized; with the aim of knowing the students' perception of methodologies and contents used during the week, their effectiveness to demonstrate the role of the obstetric professional and to motivate the continuity of first semester students in the career, a cross-sectional study was carried out descriptive and analytical through a Likert scale survey, applied to the students who attended the activities carried out during the week and the use of ordinal Bayesian models. The results of the students' perception show positive qualifications: forum with intervention by experts $90.5 \%$, keynote presentation $89.4 \%$, open house $83.6 \%$, sociodrama $90 \%$ and simulation $93.1 \%$. $90.4 \%$ of undergraduate students indicated that the topics presented demonstrated the professional role, while $84.3 \%$ of first semester students said they felt motivated to continue their studies within the career. It is concluded that the perception of the methodologies used in the week of learning results presentation is positive and through them it was possible to demonstrate the professional role and encourage motivation in first semester students to continue their training in the career.
\end{abstract}

Keywords - Medical education, undergraduate, motivation, didactics.

\title{
INTRODUCCIÓN
}

Ceis de cada diez estudiantes que ingresan a la universidad culminan de manera exitosa su formación Ny obtienen el título profesional, por otro lado se ha establecido que cerca del $30 \%$ de las deserciones obedecen a factores académicos(Buentello Martínez, 2013)(Sinchi \& Gómez Ceballos, 2018). Uno de los factores analizados dentro del aspecto académico es el ingreso de los estudiantes a una carrera no acorde a su proyecto de vida y tomando en cuenta que la mayor cifra de abandono de los estudios universitarios se produce en el primer nivel de formación, los docentes y autoridades deben establecer planes y programas dirigidos a promocionar la importancia del rol profesional dentro de la sociedad actual y motivar a los estudiantes a continuar su formación dentro de las carreras, para asegurar su permanencia en el sistema de educación superior. (Mares, Rocha, Rivas, \& Rueda, 2012).

\section{DESARROLLO}

Entre los indicadores que se toman en cuenta dentro de la evaluación, acreditación y aseguramiento de la calidad de educación en una institución universitaria está la tasa de retención, por tanto la creación de estrategias que mejoren la inclinación de los estudiantes para continuar su formación dentro de la carrera que inician, es una de las labores más relevantes(Frutos, 2017). 
Se debe tomar en cuenta que en el Ecuador, el ingreso de los estudiantes a la universidad se realiza a través de un programa de asignación de cupos acorde al segundo inciso del artículo 356 de la Constitución que señala “(...) el ingreso a las instituciones públicas de educación superior se regulará a través de un sistema de nivelación y admisión definido en la ley” (SENESCYT, 2019), a su vez el artículo 81 de la Ley Orgánica de Educación Superior indica que: “(...) El sistema se rige por los principios de méritos, igualdad de oportunidades y libertad de elección de carrera o carreras e institución (LOES, 2010), conforme a estos artículos los estudiantes en su postulación deben elegir cinco opciones de carrera y colocarlas en orden de prioridad, de tal manera que en muchos casos obtienen el cupo de ingreso para una carrera que no fue su primera elección. En un estudio previo a pesar de que el 87,5\% de estudiantes que cursó el preuniversitario para la carrera de Obstetricia dijo conocer el perfil profesional, solo el $28,6 \%$ postuló su ingreso a la carrera como primera prioridad; por otro lado el 60,7\% indicó tener la intención de realizar cambio de carrera (Toapanta-Pinta, FonsecaDávila, Ruano-Fonseca, \& Vasco-Morales, 2019). Situación que junto a otras características encontradas en el perfil de ingreso de los estudiantes creó una gran responsabilidad en autoridades y docentes para generar planes y estrategias que mejoren la afinidad de los estudiantes hacia las competencias de la profesión y que garanticen el buen desempeño académico a través de metodologías innovadoras.

Se plantea entonces la necesidad de que los estudiantes conozcan a fondo el perfil profesional o las competencias de la carrera que se encuentran cursando y por tanto la importancia de la misma en la sociedad actual, sobre todo en los estudiantes de primer semestre ya que según múltiples estudios son quienes presentan la mayor tasa de abandono de la carrera (Sinchi \& Gómez Ceballos, 2018) (Buentello Martinez, 2013) (Buentello Martínez, 2013). Para demostrar la importancia del perfil profesional se organizó la semana de exposición de resultados de aprendizaje de fin de semestre con la participación de alumnos de primero a sexto nivel de formación, quienes junto a los docentes escogieron y prepararon la modalidad de presentación (exposición magistral, foro con intervención de expertos, casa abierta, sociodrama y escenario de simulación); no hubo participación de estudiantes de séptimo y octavo semestres puesto que se encontraban cumpliendo el año de internado rotativo obligatorio.

Todo recurso didáctico innovador debe ser evaluado principalmente por los estudiantes quienes son el centro del proceso de enseñanza-aprendizaje para establecer estrategias de mejora, por tanto como parte de la autoevaluación una vez terminadas las actividades de la semana de exposición de resultados de aprendizaje fin de semestre del periodo académico septiembre 2019 a febrero 2020, se aplicó una encuesta validada según juicio de expertos de forma anónima y voluntaria a los estudiantes de la carrera (primero a sexto semestres).

La encuesta fue construida en escala Likert ya que se ha constituido como una de las principales herramientas de evaluación de la percepción de los estudiantes respecto a los programas y estrategias educativas(Matas, 2018). Cada estudiante eligió la opción que mejor refleja su punto de vista respecto a: 1) las modalidades de presentación que se utilizaron durante la semana: exposición magistral, foro con intervención de expertos, casa abierta, sociodrama y escenario de simulación, donde 1=muy inadecuado, 2=inadecuado, 3=poco adecuado, 4=adecuado, 5=muy adecuado, y 2) preguntas referentes a: ¿Las diferentes modalidades y contenidos expuestos durante la semana demostraron el verdadero rol del profesional de Obstetricia y su importancia en el sistema de salud? y ¿Las actividades y contenidos presentados durante la semana motivaron a continuar mis estudios dentro de la carrera de Obstetricia?, donde 1=totalmente en desacuerdo, 2=en desacuerdo, 3=neutral, 4=de acuerdo, 5= completamente de acuerdo. 
El total de encuestas fue de 269 y los resultados descriptivos fueron presentados a las autoridades; posteriormente se solicitó autorización al Consejo de Carrera de Obstetricia para uso de la base de datos y la realización de un análisis a través de modelos ordinales a pesar que en la mayoría de casos los autores utilizan modelos métricos, ya que por la naturaleza del cuestionario pueden producirse errores en la interpretación de los resultados (Bryer \& Speerschneider, 2016).

Se presenta entonces, un estudio transversal descriptivo y analítico en fuente secundaria, con el objetivo de conocer la percepción de los estudiantes de la carrera sobre las metodologías y contenidos utilizados en la semana de exposición de los resultados de aprendizaje obtenidos por alumnos que cursaron los diferentes niveles de formación durante el periodo académico y su eficacia para demostrar el rol del profesional de obstetricia y su importancia en el sistema de salud así como para motivar a los estudiantes de primer semestre a continuar sus estudios dentro de la carrera a través de modelos Bayesianos ordinales.

\section{Metodología}

\section{Análisis estadístico}

En el análisis se incluyó solo a 189 estudiantes (70,3\%) que corresponden a quienes asistieron a todas las actividades realizadas durante la semana. A través de estadística Bayesiana la misma que tiene como base los métodos simulación de Monte Carlo mediante las Cadenas de Markov(MCMC), que generan una gran muestra de valores de la distribución a posterior (para más detalles del modelo Bayesiano consultar a los autores Kruschke, Achar y cols) (Achar, Coelho Barros, Molina de Souza, \& Martinez, 2019), se realizó el cálculo con 500 pasos de adaptación, 1000 se quemaron y se salvó 24000 pasos. La convergencia de las cadenas fue confirmada con la prueba de Heidelberger y Welch y en la interpretación de los resultados, se tomó en cuenta el Intervalo de Densidad de Probabilidad más alta (HDI), es decir el rango de los resultados incluidos en el 95\% de los valores más creíbles (este parámetro es equivalente al intervalo de confianza al 95\% utilizado en modelos de estadística frecuentista).

\section{RESULTADOS}

De los 189 estudiantes incluidos en el análisis: El 27\% fueron de primero, el 15,9\% de segundo, el $12,12 \%$ de tercero, el $24,3 \%$ cuarto, el $12,12 \%$ de quinto y el $8,5 \%$ de sexto semestre.

En relación con las modalidades de presentación se tomaron como calificaciones positivas las correspondientes a: adecuado y muy adecuado. En la figura 1 se observa los porcentajes de calificaciones positivas. 


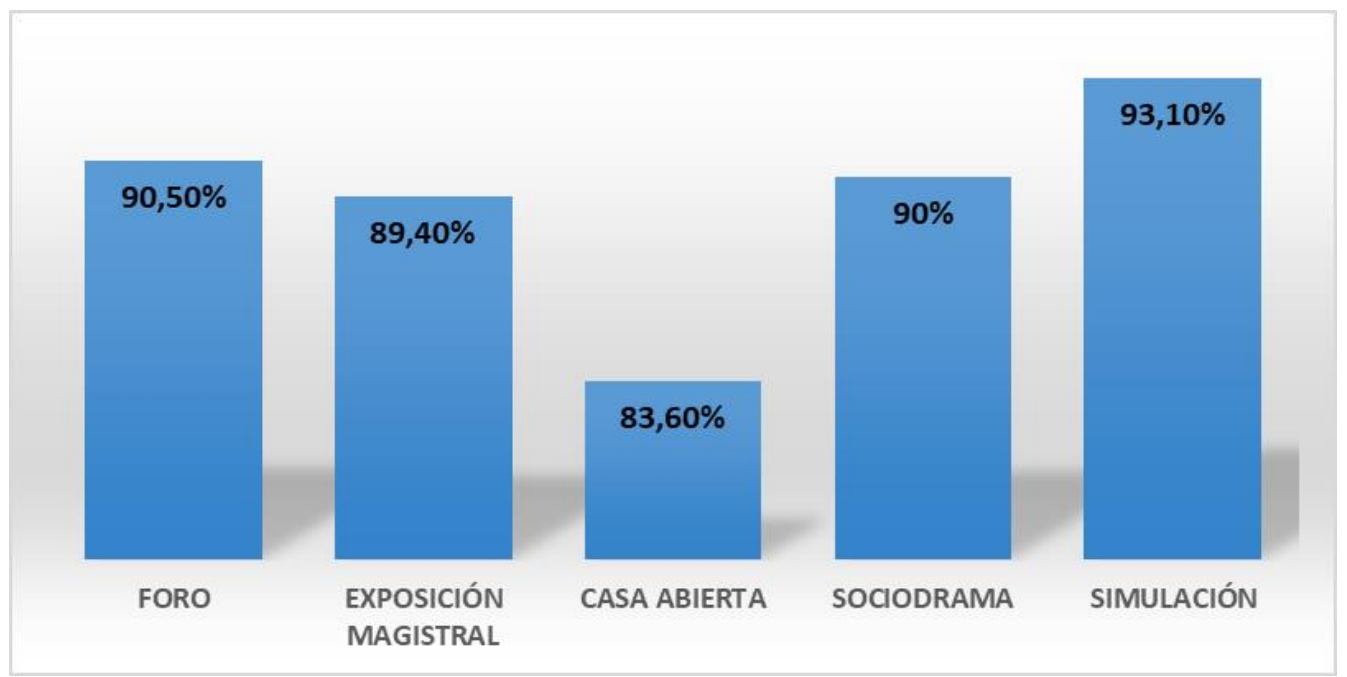

Figura 1. Porcentajes de calificaciones positivas (adecuado y muy adecuado) respecto a las diferentes modalidades utilizadas para presentación de resultados de aprendizaje

La presentación en modalidad de foro con intervención de expertos y escenario de simulación mostró diferencia estadísticamente significativa $\mathrm{p}>0,05$.

En la percepción de los estudiantes respecto a las preguntas:

1. ¿Las diferentes modalidades y contenidos expuestos durante la semana me demostraron el verdadero rol del profesional de obstetricia y su importancia en el sistema de salud? el 90,4\% de estudiantes de la carrera contestó estar de acuerdo y completamente de acuerdo.

2. ¿Las actividades y contenidos presentados durante la semana le motivaron a continuar sus estudios dentro de la carrera de obstetricia? el 84,3\% de estudiantes de primer semestre indicó estar de acuerdo y completamente de acuerdo.

Al comparar la percepción entre los estudiantes de los diferentes niveles de formación, en el análisis Bayesiano se encontró que para los estudiantes de primer semestre todas las metodologías y contenidos utilizados para exponer los resultados de aprendizaje fueron novedosas (Figura 2) 


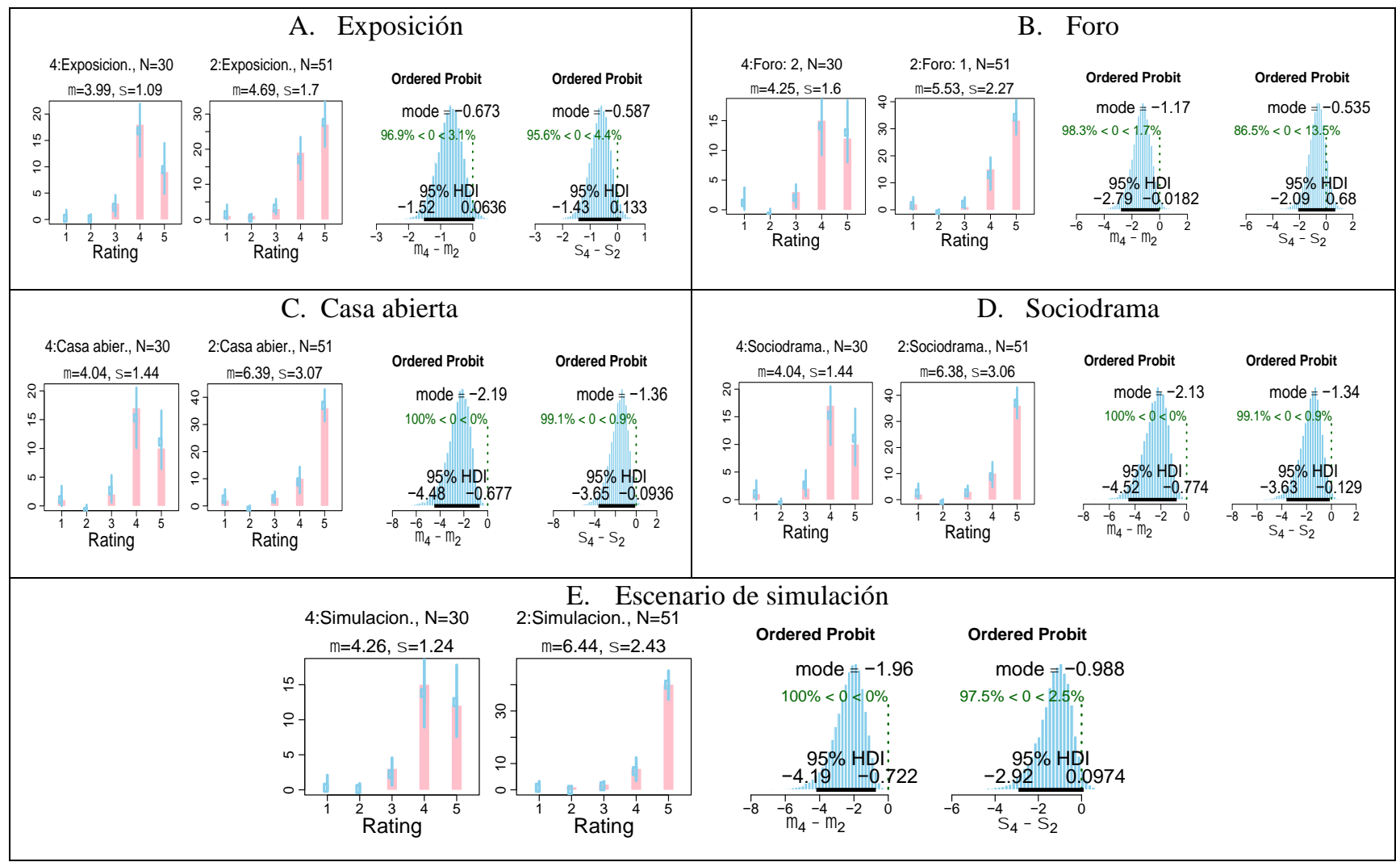

Figura 2. Comparación de la percepción entre estudiantes de primero $(\mathrm{N}=51)$ y segundo $(\mathrm{N}=30)$ respecto a las metodologías y contenidos. En los recuadros se representa la distribución de los valores de simulación vía MCMC, en la parte superior de los recuadros consta el valor de la media $(\mu)$ y el valor del desvió estándar $(\sigma)$.

En histogramas se representa los valores modales para $\mu$ у $\sigma$.

La misma tendencia se encuentra al comparar la percepción de los estudiantes de primero con tercero, cuarto, quinto y sexto semestres.

La figura 3 muestra la diferencia de medias entre la percepción de los estudiantes de primero y segundo semestre respecto a las modalidades y contenidos de la exposición de resultados de aprendizaje en la demostración del rol del profesional de obstetricia y su importancia en el sistema de salud, así como respecto a la motivación para continuar sus estudios dentro de la carrera.

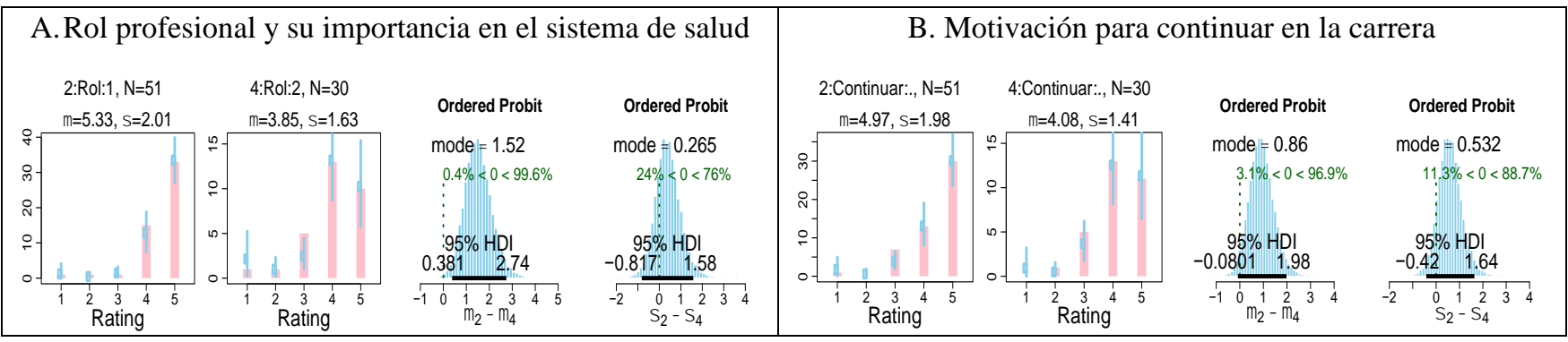

Figura 3. Comparación de la percepción entre estudiantes de primero $(\mathrm{N}=51)$ y segundo $(\mathrm{N}=30)$ A. Demostración del rol profesional y su importancia en el sistema de salud B. Motivación para continuar en la carrera. En los recuadros se representa la distribución de los valores de simulación vía MCMC, en la parte superior de los recuadros consta el valor de la media $(\mu)$ y el valor del desvió estándar $(\sigma)$. En histogramas se representa los valores modales para $\mu$ y $\sigma$ 
Al comparar con los resultados de la percepción de los estudiantes de primero con los de tercero a sexto semestre en las dos preguntas se mantiene la misma tendencia.

\section{DiSCUSIÓN}

Tomando en cuenta que las actividades realizadas durante la semana fueron planificadas como una estrategia de aprendizaje cooperativo que promueva la comprensión del rol del profesional de obstetricia y su importancia en el sistema de salud a través de la presentación de los resultados de aprendizaje por los mismos estudiantes de cada nivel de instrucción, la percepción de los alumnos respecto a las diferentes modalidades utilizadas muestra resultados alentadores. Está descrito que las estrategias utilizadas determinan el nivel de motivación de los estudiantes(Zambrano Córdova, 2019); las dos metodologías que mostraron diferencia significativa fueron el foro con intervención de expertos y el escenario de simulación.

Respecto al foro con intervención de expertos, al ser una variante de la discusión de grupos o el denominado debate donde todos los asistentes pueden participar en el análisis y discusión del tema de interés a través de la exposición de ideas y opiniones así como la formulación de preguntas, que permiten profundizar el conocimiento y con ello mejorar el proceso de enseñanza-aprendizaje a través de la reflexión conjunta(Hernández \& Guárate, 2017) ha mostrado tener un impacto positivo para promover la motivación, autoestima, compañerismo y respeto hacia los demás, por tanto es considerada una herramienta útil para la adquisición de conocimientos(Hernández Hita, Arias Mancebo, Visiers Jiménez, \& Peña Otero, 2018).

La simulación de escenarios constituye una técnica que estimula la motivación para lograr aprendizajes significativos a través de la representación operante de un modelo real, en el cual el estudiante actúa en un entorno simulado con el objetivo de practicar y desarrollar capacidades de acción y de toma de decisiones relacionadas con situaciones de la vida real (Hernández \& Guárate, 2017); se propone que la exposición temprana a los pacientes simulados fomenta el interés de los estudiantes por el aprendizaje(Quintero, 2012). A través de una revisión sistemática se ha establecido que la simulación en el aprendizaje del área obstétrica es una herramienta de alto nivel, que brinda la oportunidad de adquirir mayores habilidades en el entorno educativo, y posteriormente en el desempeño profesional. (Yucel, Hawley, Terzioglu, \& Bogossian, 2020)

El sociodrama es considerada como una metodología eficaz de comunicación didáctica, utilizada para representar emociones dentro una escena real o imaginaria, en la cual los actores, en este caso los estudiantes desempeñan determinado rol dentro de una situación que tiene inicio, desarrollo y desenlace, sin embargo además de la creatividad de los presentadores, requiere de observación y análisis por parte de los espectadores(Hernández \& Guárate, 2017), razón por la cual su desarrollo representa un verdadero reto en la promoción del pensamiento crítico(Zambrano Córdova, 2019). Los educadores reconocen la importancia de incluir en la educación de las áreas de la salud las artes liberales, donde el sociodrama se muestra muy útil en el aprendizaje interactivo y reflexivo puesto que permite el empoderamiento de los estudiantes como protagonistas de su propio aprendizaje y el de sus compañeros para el desarrollo profesional como agentes de cambio en búsqueda de la justicia social.(de Carvalho et al., 2020) 
En referencia a las técnica de exposición, y casa abierta que mostraron menor proporción de resultados positivos; probablemente sus características de presentación oral de manera estructurada y lógica con variaciones acordes al propósito del estudio de forma unidireccional donde la audiencia es receptora de la información(Hernández \& Guárate, 2017) proporcionaron menores porcentajes positivos en la percepción de los estudiantes, esto lleva a reflexionar acerca de la necesidad de evolucionar conforme las demandas de educación actual e implementar actividades más innovadoras que refuercen el interés y entusiasmo hacia la profesión y que brinden al estudiante un rol más activo y participativo(Zambrano Córdova, 2019).

La percepción de los estudiantes de primer semestre mostró valores positivos en mayor proporción que los estudiantes de todos los niveles superiores donde los resultados pueden verse influenciados debido a que apenas están empezando su formación. A través de las actividades realizadas se logró mayor motivación que justamente se constituye como la directriz para el buen desempeño académico de los universitarios, dado que su identificación con el perfil profesional incentiva la disponibilidad de continuar con sus estudios. En un estudio previo (cuando los estudiantes que ahora completaron el primer semestre cursaron el preuniversitario para la carrera) se encontró que solo el 28,6\% postuló para la carrera como primera prioridad, además hubo diferencia significativa entre prioridad de la carrera en la postulación para el ingreso y el conocimiento del perfil profesional y de la importancia de la carrera, de la misma manera con la intención de realizar cambio de carrera(Toapanta-Pinta et al., 2019); está claramente establecido que aquellos estudiantes que no solicitaron el ingreso a una carrera como su primera opción tienen poca motivación al iniciar sus estudios en un área que es la segunda, tercera, cuarta o quinta alternativa en su proyecto de vida(Mares et al., 2012), por otro lado el desconocimiento del perfil profesional constituye un factor negativo(Sinchi \& Gómez Ceballos, 2018), por el contrario la percepción del rol dentro de un perfil profesional impacta de manera positiva como fuente de motivación en el desenvolvimiento académico de los estudiantes y por tanto la continuidad y culminación de su formación universitaria(Herrera \& Burgoa, 2013).

En este estudio el 90,4\% de los estudiantes indicó que las diferentes modalidades y contenidos expuestos durante la semana demostraron el rol del profesional de obstetricia y su importancia en el sistema de salud; y el 84,3\% de estudiantes de primer semestre consideró que estos le motivaron a continuar sus estudios dentro de la carrera de obstetricia, a pesar que en un estudio previo el $60,7 \%$ indicó que realizaría cambio de carrera(Toapanta-Pinta et al., 2019). En el fomento del interés de los futuros profesionales por las competencias de las diferentes áreas de la salud juega un rol importante la implementación de condiciones pedagógicas, destinadas a desarrollar la motivación positiva hacia la formación en el área de estudio; la elección y el ingreso a una determinada profesión en la mayoría de los estudiantes depende de motivos sociales, pero al ingresar a una carrera si las asignaturas muestran un bajo nivel de enfoque en el aprendizaje dirigido hacia el perfil profesional aumentarán las cifras de deserción por falta de afinidad de los estudiantes hacia las competencias; por el contrario si se implementan metodologías que permitan la integración de conocimientos, habilidades y destrezas de varias asignaturas y de los diferentes niveles de formación se prepara a los estudiantes para cursar materias académicas en el siguiente nivel de estudio (Morokhovets et al., 2019); pues con la exposición de los resultados de aprendizaje por los mismos estudiantes, se logró una mayor motivación a continuar la formación académica en la carrera. 


\section{CONCLUSIONES}

Las diferentes metodologías utilizadas para la exposición de resultados de aprendizaje al finalizar el semestre fueron calificadas como adecuadas y muy adecuadas por la mayoría de los estudiantes, y lograron mostrar el rol del profesional de Obstetricia y su importancia dentro de la sociedad actual, además sirvieron para motivar a los estudiantes de primer semestre a continuar su formación dentro de la Carrera.

\section{REFERENCIAS BIBLIOGRÁFICAS}

- Achar, J. A., Coelho Barros, E. A., Molina de Souza, R., \& Martinez, E. Z. (2019). Uma introdução aos métodos bayesianos aplicados à análise de dados (Cia do eBo; Equipe Cia do eBook, Ed.). Timburi/SP: EDITORA CIA DO EBOOK.

- Bryer, J., \& Speerschneider, K. (2016). Analysis and Visualization Likert Items. The Comprehensive $R$ Archive Network, p. 17. Retrieved from https://cran.r-project.org/web/packages/likert/likert.pdf

- Buentello Martinez, C. P. (2013). Deserción Escolar, Factores Que Determinan El Abandono De La Carrera Profesional. Universidad Aútonoma De Sinaloa Facultad De Contaduría Y Administración, (214), 1-25.

- de Carvalho, F. M. A., Ledubino, A., Frutuoso, L., da Silva, W. J., Jaarsma, D., Helmich, E., \& Strazzacappa, M. (2020). Medical Education Empowered by Theater (MEET). Academic Medicine: Journal of the Association of American Medical Colleges. Retrieved from https://www.ncbi.nlm.nih.gov/pubmed/32134785

- Frutos, C. (2017). Análisis de la tasa de retención y su incidencia en la detección de patrones de deserción estudiantil en la Universidad Técnica de Ambato. Retrieved from http://repositorio.uta.edu.ec/jspui/handle/123456789/25797

- Hernández, C. A., \& Guárate, A. Y. (2017). Modelos didácticos: Para situaciones y contextos de $\begin{array}{llll}\text { aprendizaje (Vol. 146). } & \text { Retrieved }\end{array}$ https://books.google.es/books?hl=es\&lr=\&id=cFQ1DwAAQBAJ\&oi=fnd\&pg=PA80\&dq=Modelos +didacticos:+para+situaciones+y+contexto+de+aprendizaje\&ots=_w7NJgr8iP\&sig=jeH5PAjFWql $\mathrm{H} 6 \mathrm{HbVtVK} 909 \mathrm{wCD} 5 \mathrm{U} \# \mathrm{v}=$ onepage \&q=Modelos didacticos\%3A para situaciones y contexto de aprend

- Hernández Hita, N., Arias Mancebo, C., Visiers Jiménez, L., \& Peña Otero, D. (2018). El foro de discusión como herramienta docente en práticas tuteladas del grado en enfermeria. Revista de Enfermagem Referência, IV(17). https://doi.org/https://doi.org/10.12707/RIV17101

- Herrera, E. F., \& Burgoa, T. (2013). La Importancia del Perfil Vocacional en la Visión del Estudiante Universitario: Caso de Estudio en Facultades de la Universidad Autónoma de Nuevo León, UANL. International Journal of Good Conscience, 8(2), 23-47. Retrieved from http://www.spentamexico.org/v8-n2/A2.8(2)23-47.pdf 
- LOES. (2010). Ley Orgánica De Educación Superior Ecuador. Ley Orgánica de Educación Superior Del Ecuador, 1-63. Retrieved from http://educaciondecalidad.ec/leyes-sistema/ley-educacionsuperior-loes.html

- Mares, G., Rocha, H., Rivas, O., Cabrera, R., Tovar, J., \& Medina, L. (2012). Identificación de factores vinculados con la deserción y la trayectoria académica de los estudiantes de Psicología en la FES IZTACALA. Enseñanza E Investigación En Psicología, 17(1), 189-207. Retrieved from http://www.cneip.org/documentos/12.pdf

- Matas, A. (2018). Diseño del formato de escalas tipo Likert: un estado de la cuestión. Revista Electrónica de Investigación Educativa, 20(1), 38-47. Retrieved from https://doi.org/10.24320/redie.2018.20.1.1347\%0A

- Morokhovets, H. Y., Uvarkina, O. V, Bieliaieva, O. M., Lysanets, Y. V, Senkevych, H. A., \& Stetsenko, S. A. (2019). DEVELOPMENT OF MOTIVATION TOWARDS EDUCATION IN MEDICAL STUDENTS ROZWÓJ MOTYWACJI DO NAUKI U STUDENTÓW MEDYCYNY. Wiad Lek, 72(1), 7-11. Retrieved from https://www.ncbi.nlm.nih.gov/pubmed/30796853

- Quintero, G. A. (2012). Educación Médica: Diseño e implementación de un currículo basado en resultados de aprendizaje. from https://books.google.es/books?hl=es\&lr=\&id=8KGkDwAAQBAJ\&oi=fnd\&pg=PR3\&dq=Quinter+ Gustavo,+SIMULACIÓN:+Educación+médica:+Diseño+e+implementación+de+un+currículo+basa do+en+resultados+del+aprendizaje\&ots=VWYYAN6Tze\&sig=rCYheJQ11K9zT5BMzvL9kd1ZT6 $\mathrm{Y} \# \mathrm{v}=$ onepage\&q

- SENESCYT. (2019). REGLAMENTO-SNNA, Acuerdo-N 2019-030 (p. 26). p. 26. Retrieved from http://admision.senescyt.gob.ec/media/2019/05/REGLAMENTO-SNNA_-Acuerdo-N---2019030.pdf

- Sinchi, E. R., \& Gómez Ceballos, G. P. (2018). Acceso y deserción en las universidades. $\begin{array}{lllll}\text { Alternativas de } \quad \text { financiamiento. } & \text { Alteridad, }\end{array}$ https://doi.org/10.17163/alt.v14n2.2018.10

- Toapanta-Pinta, P., Fonseca-Dávila, L., Ruano-Fonseca, L., \& Vasco-Morales, S. (2019). Perfil de los estudiantes que ingresan a la Carrera de Obstetricia-Universidad Central del Ecuador. Magazine de Las Ciencias, $\quad 4(4 \quad$ (Octubre-Dicembre), $\quad 127-136$. https://doi.org/https://doi.org/10.5281/zenodo.3605190

- Yucel, C., Hawley, G., Terzioglu, F., \& Bogossian, F. (2020). The Effectiveness of SimulationBased Team Training in Obstetrics Emergencies for Improving Technical Skills: A Systematic Review. Simulation in Healthcare: Journal of the Society for Simulation in Healthcare. Retrieved from https://www.ncbi.nlm.nih.gov/pubmed/32168287

- Zambrano Córdova, M. M. (2019). Guía metodológica de la estrategia del sociodrama como herramienta de enseñanza del idioma inglés en los estudiantes del cuarto nivel de la Universidad Estatal del Sur de Manabí. (Universidad Católica de Santiago de Guayaquil). Retrieved from http://192.188.52.94/handle/3317/14148 